\title{
Aplicación del derecho y los casos Noara y Jaffa Cakes (en diálogo con Luís Duarte d'Almeida)
}

\author{
The Application of Law and the Noara and \\ Jaffa Cakes cases (A Conversation with Luis \\ Duarte d'Almeida) \\ José Juan Moreso*
}

The fact that a law applies to someone always consists in more than a semantic relation; it is not merely that a person falls under some description contained in the law.

Bernard Williams (1985, p. 191)

Recepción: 30/09/2021

Evaluación: 05/10/2021

Aceptación final: 12/10/2021

Resumen: En este trabajo se comentan algunas tesis sobre la aplicación del derecho de Luís Duarte d'Almeida. Dos son las tesis discutidas: 1) Que la concepción deductivista no reconstruye adecuadamente el razonamiento judicial y 2) que en dicho razonamiento basta con establecer que una determinada norma $\mathrm{N}$ es aplicable a un caso c. Se intenta mostrar que hay un modo de rescatar la concepción deductivista del razonamiento de los jueces y que dicha concepción contribuye a desvelar lo que significa que una norma $\mathrm{N}$ es aplicable a un caso $\mathrm{c}$.

Palabras clave: aplicación del derecho, razonamiento judicial, inferencia lógica.

Catedrático de Filosofía del Derecho, Universitat Pompeu Fabra, Barcelona, España. Correo electrónico: josejuan.moreso@upf.edu 
José Juan Moreso

\begin{abstract}
In this work some of Luís Duarte d'Almeida's theses on the application of law are commented. Two are the disputed theses: 1) That the deductivist conception does not adequately reconstruct judicial reasoning and 2) that in judicial reasoning it is enough to establish that a certain norm $\mathrm{N}$ is applicable to a case $\mathrm{c}$. An attempt is made to show that there is a way to rescue the deductivist conception of judges' reasoning and that this conception contributes to revealing what it means that a norm $\mathrm{N}$ is applicable to a case $\mathrm{c}$.
\end{abstract}

Keywords: application of law, judicial reasoning, logical inference.

\title{
1. Introducción
}

Luís Duarte d’Almeida anunciaba ya sus heterodoxos puntos de vista sobre la aplicación del derecho en su importante libro (2015) para nuestra noción del razonamiento jurídico, pero su concepción de la aplicación del derecho ha sido desarrollada en dos trabajos seminales (Duarte d'Almeida, 2019, 2021), el segundo de los cuales comentamos en este número de Discusiones. En el primero argumenta contra la idea, ampliamente aceptada, de que aplicar el derecho es una operación deductiva, que consiste en mostrar que el contenido de la decisión judicial se deriva lógicamente del contenido de una norma aplicable y de una proposición verdadera que describe los hechos del caso, lo que se conoce como silogismo judicial. En el segundo, la pars construens de su enfoque, presenta su propia concepción.

La capacidad de Luís de diseccionar los problemas y de resolverlos con agudeza y parsimonia es asombrosa. Es mucho lo que aprendemos leyéndole. El modo en que muestra (en Duarte d'Almeida, 2019) las deficiencias y oscuridades en la presentación del modelo deductivista de razonamiento judicial por parte de sus defensores, entre los que se hallan los más perspicuos de los filósofos del derecho, es refrescante y clarificador. ${ }^{1}$

1 En castellano hay otro autor, Rafael Hernández Marín (por ejemplo, 2019), que también ha criticado duramente la concepción deductiva del razonamiento judicial. Sus argumentos se solapan de vez en cuando con los de Duarte d'Almeida, pero — según creo- se alejan en lo principal. 
Aplicación del derecho y los casos Noara y Jaffa Cakes (en diálogo...

En estos comentarios trataré de mostrar, en primer lugar, que un defensor de la concepción tradicional - como yo lo soy $-{ }^{2}$ puede resistir las críticas de la pars destruens de Luís y, en segundo lugar, trataré también de argüir un modo en el cual el núcleo de la concepción deductiva puede incorporarse a la propia concepción de Luís manteniendo su integridad. Y lo haré valiéndome de dos casos resueltos en sede judicial, uno en España, el caso Noara ${ }^{3}$ y otro en el Reino Unido, el caso Jaffa Cakes. ${ }^{4}$

\section{El caso Noara}

Comencemos con el caso Noara. ${ }^{5}$ Noara era un bebé que necesitaba con urgencia un trasplante de hígado, con el riesgo de perder la vida. Entre personas compatibles, el trasplante es posible entre vivos porque solo se precisa una pequeña parte del hígado del donante. Por fortuna, la propia madre de Noara, Rocío, era compatible, y — como se puede comprender- deseaba ardientemente ser su donante. Ahora bien, Rocío tenía solo 16 años y el art. 4a) de la Ley 30/1979, de 27 de octubre, sobre extracción y trasplante de órganos establece: "la obtención de órganos procedentes de un donante vivo, para su ulterior injerto o implantación en otra persona, podrá realizarse si se cumplen los siguientes requisitos: a) que el donante sea mayor de edad". Y una más que plausible interpretación de esta norma implica que la donación de un órgano por parte de un menor de edad está prohibida.

El caso Noara se resolvió felizmente con el Auto referido en la nota 3, en donde la jueza de Sevilla decidió autorizar el trasplante apelando ana

2 Y él tiene la amabilidad de reconocerme en este contexto, refiriéndose como ejemplo de este punto de vista a Moreso y Chilovi (2018, p. 495) en Duarte d'Almeida (2021, p. 365 y nota 2).

3 Auto (785/07, de dieciocho de octubre) del Juzgado de Primera Instancia de Sevilla n.17. https://www.rexurga.es/descarga/J\%5B1\%5D.Primera\%20Instancia\%20Sevilla\%20\%2898407\%29.pdf

4 United Biscuits (UK) Ltd (No. 2). [1991] BVC 818, 21 August 1991. https://app.vlex. com/\#vid/806616417

5 Fue García Figueroa el que nos llamó la atención sobre este caso, usándolo para defender una concepción principialista del razonamiento jurídico, según la cual el razonamiento jurídico es una cuestión de principios más bien que de reglas (García Figueroa, 2009, pp. 152-153 y 2012, pp. 126-128). 
lógicamente a que, en los supuestos de esterilización, de acuerdo con la legislación y la jurisprudencia, el consentimiento del menor puede suplirse a través de la solicitud del tutor legal (la madre de Rocío en este caso), la conformidad consciente del menor, el informe de especialistas y la intervención del Ministerio Fiscal, que finalizan en una autorización judicial que suple el consentimiento de la menor.

No hay en el texto del Auto argumento ninguno para mostrar que la disposición contenida en el art. 4a) de la Ley 30/1979 deja de ser aplicada, aunque hay una apelación a la analogía de los supuestos de esterilización, pero solo para sostener la necesidad de la autorización judicial en este caso. Sin embargo, la jueza parece suponer que autorizando el trasplante en este caso está aplicando el art. 4 de la Ley 30/1979 a pesar de que Rocío, la donante, es menor de edad.

Y este es el argumento crucial, precisamente, que Luís usa contra la concepción deductivista, el deductivismo de las reglas como prefiere el autor $(2019,337)$. ¿Cuál podría ser la reconstrucción del argumento en este caso? Algo como:

(1) Todas las personas mayores de edad, en posesión de sus facultades mentales, debidamente informadas y prestando su consentimiento de manera libre y consciente pueden donar órganos para su injerto o implantación en otra persona.

(2) Rocío es una menor de edad, en posesión de sus facultades mentales, debidamente informada y prestando su consentimiento de manera libre y consciente, dispuesta a donar parte de su hígado para ser trasplantado a su hija Noara.

Por lo tanto, de (1) y (2),

(3) Rocío está autorizada a donar parte de su hígado a su hija Noara.

Y el problema ahora es obvio: ${ }^{6}$ (3) no se deriva de (1) y (2). (1) es una oración universal condicional (algo como "Para toda persona $\mathrm{x}$, tal que $\mathrm{x}$ sea F, G y H, entonces X tiene permitido hacer A"), (2) dice algo como

6 Luís usa un caso distinto pero con efectos semejantes, $R v$ Luffe (1807) 8 East 193 (Duarte d’Almeida 2019, 351-359). 
Aplicación del derecho y los casos Noara y Jaffa Cakes (en diálogo...

"n no es F, es G y es H". Con estas dos premisas es imposible derivar (3). Tenemos un condicional universal, pero en la segunda premisa tenemos una oración que niega el antecedente de (1) y no podemos aplicar, por lo tanto, el modus ponens.

Luís explora dos posibles respuestas de los defensores del modelo, consistentes en cambiar la premisa mayor y cambiar la premisa menor respectivamente. Para la primera estrategia, se trataría de excluir la mayoría de edad de la premisa (1) y entonces podríamos obtener (3). En la segunda, en cambio, la premisa menor diría que Rocío es mayor de edad. Según Luís, no es esto lo que suelen hacer los jueces. Si hicieran lo primero no podrían decir que aplican la disposición jurídica en cuestión porque estarían aplicando otra disposición. Si hicieran lo segundo el argumento sería formalmente válido, pero no sería sólido, porque algo como “(2’) Rocío es mayor de edad, en posesión de sus facultades mentales, debidamente informada y prestando su consentimiento de manera libre y consciente, dispuesta a donar parte de su hígado para ser trasplantado a su hija Noara" es falso, Rocío tiene solo 16 años. Luís piensa que esta situación resulta fatal para el deductivismo de las reglas, que se halla en un callejón sin salida, porque no puede, a la vez, sostener que la jueza resolvió (3) y que (3) es una aplicación de la disposición en cuestión porque se deriva de (1) y (2).

Suponiendo que he reconstruido bien su forma de razonar, es claro que es un argumento poderoso. El fallo, la parte dispositiva de la sentencia, es el siguiente (los nombres están cambiados en ella para preservar su anonimato):

DEBO AUTORIZAR Y AUTORIZO a Gabriela a la donación de hígado solicitada para su hija Verónica, quedando así suplida su falta de capacidad, integrándose el consentimiento de la misma.

Sin embargo, considero que el razonamiento de la jueza es insuficiente para mostrar lo que desea mostrar. El art. 4 de la Ley, por sí solo, no autoriza a la jueza a suplir la falta de la capacidad de la madre. Creo que la solución es correcta, conforme al derecho español, pero la jueza debería haber razonado de otro modo. Debería haber mostrado que en el derecho español también existe la causa de justificación de estado de necesidad, que está regulada en el art. 20 del Código Penal de este modo: 
José Juan Moreso

Están exentos de responsabilidad criminal:

(...)

5. ${ }^{\circ}$ El que, en estado de necesidad, para evitar un mal propio

o ajeno lesione un bien jurídico de otra persona o infrinja un deber, siempre que concurran los siguientes requisitos: Primero. Que el mal causado no sea mayor que el que se trate de evitar.

Segundo. Que la situación de necesidad no haya sido provocada intencionadamente por el sujeto.

Tercero. Que el necesitado no tenga, por su oficio o cargo, obligación de sacrificarse.

Y, a la vista de ello, razonar así: el médico que realice la extracción de la parte del hígado de la madre de Noara y la madre de Noara que lo permite, realizan un acto permitido por el derecho español, a pesar de que infringen un deber, el que impide a los menores de edad ser donantes, dado que se cumplen los tres requisitos del estado de necesidad. Es obvio, me parece, que la muerte de Noara, segura sin el trasplante, es un mal mayor que la infracción del deber del art. 4 de la Ley y también es verdad que dicha situación no ha sido provocada intencionadamente por nadie, y ni el médico, ni Noara, ni la madre de Noara tienen, por su oficio o cargo, la obligación de sacrificarse. Así funcionan siempre las causas de justificación. Y dicho razonamiento puede ser reconstruido deductivamente del siguiente modo:

(1") Todas las personas mayores de edad, en posesión de sus facultades mentales, debidamente informadas y prestando su consentimiento de manera libre y consciente pueden donar órganos para su injerto o implantación en otra persona.

(2") Todas las personas están autorizadas a infringir un deber cuando están en estado de necesidad.

(3") Rocío es una menor de edad, en posesión de sus facultades mentales, debidamente informada y prestando su consentimiento de manera libre y consciente, dispuesta a donar parte de su hígado para ser trasplantado a su hija Noara.

(4") Roció está en estado de necesidad, puesto que, si no dona parte de su hígado a Noara, entonces Noara morirá. 
Aplicación del derecho y los casos Noara y Jaffa Cakes (en diálogo...

Por lo tanto, de (1"), (2"), (3") y (4"), sí puede deductivamente obtenerse

(5”) Rocío está autorizada a donar parte de su hígado a su hija Noara.

Caer en la cuenta de que el razonamiento judicial debe considerar todas las normas aplicables a determinada situación es aquí crucial. Es obvio que mi conclusión puede controvertirse, por ejemplo, porque alguien considera que aquí no es de aplicación el estado de necesidad, pero si es - como yo creo- aplicable, entonces debe ser aplicado y el contenido de la decisión se deriva de las premisas.

En la siguiente sección trataré de mostrar que esta concepción puede ser incluida en el enfoque de Luís preservando gran parte de lo que sostiene.

\section{El caso Jaffa Cakes}

En 1991, un Tribunal inglés competente para conocer los recursos de apelación sobre decisiones de la administración acerca del impuesto del valor añadido se pronunció sobre (Value Added Tax Act 1983) si los dulces Jaffa Cakes, elaborados y comercializados por la empresa McVities, estaban exentos o no del pago de dicho impuesto. Como mis lectores sabrán, se trata de unos dulces compuestos de un bizcocho relleno de mermelada de naranja (de ahí su nombre que evoca las naranjas de Jaffa) y recubierto por una capa de chocolate negro. La cuestión jurídica surge de la cuestión de si es aplicable o no la cláusula primera de la Schedule 5 de la ley, que establece que están sujetos al tipo impositivo 0 , en su número 1, "Food of a kind used for human consumption", aunque hay una serie de ítems exceptuados, y aquí es relevante la segunda de las excepciones "Chocolates, sweets and similar confectionery (including drained, glace or crystallized fruits); and biscuits and other confectionery (not including cakes) wholly or partly covered with chocolate or some product similar in taste and appearance".

Es una regulación interesante porque muestra cuán a menudo el derecho es una cuestión de excepciones a las excepciones de las excepciones. No cabe duda que los Jaffa Cakes son un tipo de alimento usado para el consumo humano, tampoco cabe duda de que está cubierto de chocolate, 
lo que lo sujetaría al tipo estándar del IVA, pero (la excepción de la excepción) estaría exento del impuesto si fuese realmente un "cake", un pastel. $\mathrm{O}$ sea, como bien establece el Tribunal, se trata de determinar si estos dulces son pasteles (cakes, exentos del IVA) o galletas (biscuits, sujetos al IVA). El Tribunal toma en consideración algunas semejanzas de los Jaffa Cakes con las galletas, como que son de tamaño más semejante a las galletas, se comen con los dedos como las galletas, se empaquetan como ellas y aparecen en los comercios que las venden agrupados junto con las galletas y no con los pasteles. Hay, sin embargo, otros elementos que las asemejan a los pasteles, cuando pasa el tiempo al igual que los pasteles se ponen duras y resecas, mientras las galletas con el paso del tiempo se ablandan. Es más, para mostrar las semejanzas con los pasteles, la empresa productora elaboró un Jaffa Cake grande como un pastel de cumpleaños y lo aportó como prueba. Estos segundos argumentos convencieron en mayor medida al Tribunal que concluyó del siguiente modo:

Generally, I come to the conclusion that Jaffa Cakes have characteristics of cakes, and also characteristics of biscuits or non-cakes. I conclude that they have sufficient characteristics of cakes to qualify as cakes within the meaning of Sch. 5, Grp. 1, item 1. If it be relevant, I also determine that the Jaffa Cakes are not biscuits. I therefore allow the appeal. The appellants are at liberty to make an application in respect of costs. (Appeal allowed)

Voy a usar este ejemplo para explicar algunas ideas de la pars construens de Luís. ${ }^{7}$ Luís (Duarte d’Almeida, 2021, pp. 361-362) distingue muy convenientemente entre el objeto directo de la aplicación, ¿qué es aquello que se aplica?; el objeto indirecto de dicha aplicación, ¿a qué se aplica? Y, por último, cuál sea el contenido de la aplicación del derecho. Aunque en el trabajo hay relevantes cuestiones acerca de las dos primeras cuestiones: se aplican leyes, disposiciones, reglas, principios, precedentes, costumbres, doctrinas, etc., y sobre la segunda, se dice normalmente que estas pautas se aplican a casos, a los hechos del caso particular, se suele decir (y Luís creo que mi ejemplo también valdrá. 
Aplicación del derecho y los casos Noara y Jaffa Cakes (en diálogo...

aduce pertinentes consideraciones sobre la ambigüedad de la expresión "caso" en estos contextos), es a la tercera cuestión a la que básicamente está dedicado el trabajo.

En relación con ella, el autor distingue muy convenientemente entre la aplicación inferencial del derecho y la aplicación pragmática del derecho.

En el caso Jaffa Cakes, el objeto directo de la aplicación es la Schedule 5 de la Value Added Tax Act 1983, junto con su excepción segunda y el objeto indirecto está referido fundamentalmente a la clasificación de estos dulces o bien como pasteles o bien como galletas. La aplicación inferencial del derecho es un proceso de justificación que cualquiera de nosotros puede realizar y que habitualmente también realizan los jueces, para determinar el contenido del derecho en una cuestión particular. La aplicación pragmática del derecho ya no es una inferencia, sino que es un acto que resuelve una cuestión dirimida, un acto que solo puede ser llevado a cabo de manera exitosa por la persona competente para resolverlo, habitualmente un juez o un Tribunal. Al final del fallo del caso Jaffa Cakes, se escribe "Appeal Allowed", que es la consecuencia performativa del acto llevado a cabo por el Tribunal.

Veamos ahora cuál es el proceso inferencial que se llevó a cabo en este caso, de acuerdo con las ideas de Luís:

(1) Si la Schedule n. 5 de la Value Added Tax Act 1983 es aplicable a la comercialización de los Jaffa Cakes, entonces dichos dulces están sujetos al tipo impositivo 0 del IVA.

(2) La Schedule n. 5 de la Value Added Tax Act 1983 es aplicable a la comercialización de los Jaffa Cakes.

Por lo tanto, de (1) y (2),

(3) Los Jaffa Cakes están sujetos al tipo impositivo 0 del IVA o, lo que es lo mismo, están exentos del pago del IVA.

La premisa (1) no es, como en la concepción deductivista, un enunciado jurídico universal de primer orden. Luís insiste aquí (2021, pp. 365356) en que el Tribunal no dice ni siquiera que los pasteles están siempre y en todos los casos exentos del impuesto, ni que las galletas están siempre y en todos los casos incluidas. Dice únicamente que los Jaffa Cakes son pasteles y que están exentos. 
José Juan Moreso

Por otro lado, como Luís bien afirma, los jueces suelen también dar argumentos para justificar su aplicación pragmática, su acto. Un argumento que podría ser reconstruido así:

(1) Si la Schedule n. 5 de la Value Added Tax Act 1983 se aplica a la comercialización de los Jaffa Cakes, entonces dichos dulces están sujetos al tipo impositivo 0 del IVA.

(2) La Schedule n. 5 de la Value Added Tax Act 1983 es aplicable a la comercialización de los Jaffa Cakes.

Por lo tanto, de (1) y (2),

(3) Los Jaffa Cakes están sujetos al tipo impositivo 0 del IVA o, lo que es lo mismo, están exentos del pago del IVA.

(4) Si los Jaffa Cakes están sujetos al tipo impositivo 0 del IVA o, lo que es lo mismo, están exentos del pago del IVA, y no hay consideraciones compensatorias ("countervailing considerations" en la versión inglesa), entonces nosotros (es decir, el Tribunal) debemos jurídicamente admitir la apelación.

(5) No hay consideraciones compensatorias.

Por lo tanto, de (3), (4) y (5),

(6) Jurídicamente debemos admitir la apelación.

Esto es, el Tribunal muestra que tiene el deber de realizar el acto de admisión de la apelación. No tengo mayores objeciones a esta reconstrucción. Y me referiré, de nuevo, fundamentalmente a la reconstrucción del modelo de razonamiento de la aplicación inferencial del derecho. Sin embargo, no me resisto a hacer notar antes de pasar a ello, aunque no puedo detenerme en este asunto ahora, que la introducción de las "countervailing considerations" en (4) y (5) guarda una analogía con la estrategia que algunos han seguido para tratar de defender algo como una vía intermedia entre el generalismo y el particularismo, como Holton (2002) introduciendo su cláusula "that's it" o MacKeever y Ridge (2006) añadiendo la premisa "and no other feature of the situation explains why". Una vía, por cierto, que Luís rechazaba en Duarte d'Almeida, con especial referencia a Holton (2015, pp. 181-183). 
Aplicación del derecho y los casos Noara y Jaffa Cakes (en diálogo...

Volvamos, entonces, a la aplicación inferencial del derecho. Llamemos al argumento con las premisas (1), (2) y (3), la inferencia jurídica (IJ). Pues bien, ¿cómo se justifica (2), la premisa que dice que una pauta es aplicable a un caso? Y, ¿por qué Luís piensa que no se justifica acudiendo a pautas generales? Creo que la respuesta se halla en estas palabras:

La aplicación del derecho es, en cierto sentido, un asunto particularista. Lo que quiero decir no es que, para cualquier conclusión correctamente alcanzada sobre la base de que una determinada disposición $\mathrm{p}$ se aplica a un determinado objeto $\mathrm{x}$, no exista un principio universal verdadero que capte todos y sólo aquellos aspectos de $\mathrm{x}$ que son relevantes para esa conclusión —un principio que podría utilizarse para construir una inferencia que se ajustara a un esquema idéntico al del silogismo jurídico. Lo que quiero decir es que nosotros $-\mathrm{y}$ los tribunales en particular- no nos comprometemos (de hecho, ni siquiera podría esperarse que lo hiciéramos) con formulaciones de principios de ese tipo como un paso necesario, típico o incluso mínimamente frecuente en nuestro razonamiento o en nuestros argumentos hacia tales conclusiones (Duarte d'Almeida, 2021, p. 369).

Es decir, según Luís, la textura de la aplicación del derecho es particularista, aunque tal vez solo por razones epistémicas, al margen de consideraciones metafísicas y metaéticas. Es en este punto en el que no estoy de acuerdo con Luís. Porque, como dice Josep Raz (1999) discutiendo el particularismo precisamente, "la inteligibilidad (de las razones) depende de la generalidad” (p. 235). Es decir, para que la premisa (2) de IJ, que dice que la pauta contenida en la Schedule n. 5 de la Value Added Tax Act 1983 es aplicable a la cuestión del pago del IVA en la comercialización de los Jaffa Cakes, sea inteligible, es necesario que se apoye en algunas razones que son de carácter general. De no ser así, premisas como (2) son totalmente opacas y no podemos evaluar su adecuación. ¿Cómo pude hacerse esto de modo compatible con el hecho de que hay razones jurídicas a favor y en contra de la aplicabilidad de una pauta y ellas no son fácilmente codificables en fórmulas canónicas universales? 
José Juan Moreso

Creo que la solución viene de la mano de lo que a veces se ha denominado un generalismo contextualista. Por ejemplo, David Wiggins (2006, p. 352) argumenta del siguiente perspicuo modo:

Let me point out that it is only with the help of contextualism that we have any evident hope of formulating moral judgments that will hold universally and for absolutely all cases (that is, for all cases that fall under description that enter into the question that is under consideration).

En diversos trabajos he tratado de articular una posición de la aplicación del derecho en esta línea (valga por todos, Moreso, 2017). Se reconoce la imposibilidad de generar pautas universales válidas para todos los casos. Sin embargo, es posible generar un universo de casos que incluya el caso en cuestión y un conjunto de propiedades relevantes que permiten formular las pautas aplicables, con carácter general, a dicho universo de casos. ${ }^{8}$ Dejo las peculiaridades del modelo para otro momento, porque lo único relevante aquí es que, en mi opinión, hay un modo de hacerse cargo de la objeción de Luís y a la vez mantener la concepción deductivista, la concepción ortodoxa.

\section{Para concluir: dos razones a favor de la concepción ortodoxa}

La concepción ortodoxa, que se remonta a Beccaria $(1764,1981)$, como bien nos recuerda Luís, ha tenido en las últimas décadas egregios representantes, con matices y acentos diversos, es claro. ${ }^{9}$ Creo que hay dos buenas asunciones, al menos, que se hallan tras la defensa de la concepción ortodoxa.

La primera es la de que, si la actividad de guiar el comportamiento de los seres humanos mediante órdenes y directivas ha de ser una actividad Atienza (2013), Ferrajoli (1989), MacCormick (1978), Wróblewski (1974). 
Aplicación del derecho y los casos Noara y Jaffa Cakes (en diálogo...

racional, entonces presupone que las autoridades que dan las órdenes no solo dan las ordenes que explícitamente comunican a sus destinatarios, sino también aquellas que están implicadas por ellas. Cuando yo le decía, en los años que era pequeña, a mi hija Júlia: "Debes lavarte los dientes todos los días antes de acostarte", también le ordenaba, implícitamente, "Debes lavarte los dientes los dientes los martes antes de acostarte". Es obvio que hay casos en que esta regla no ha de ser aplicada, por ejemplo, si se halla de excursión, ha perdido su cepillo y la pasta de dientes, y debe recorrer diez kilómetros caminando para conseguir lavárselos. Sería estúpido quien se empeñara en que la regla debe ser cumplida en este caso.

Sea como fuere, los códigos normativos, si son racionales, están clausurados mediante sus consecuencias lógicas. En palabras elegantes de Georg Henrik von Wright:

The derived commands, prohibitions and permissions of a corpus of prescriptions we could say are as much "willed" by the norm-authority as the original commands, prohibitions and permissions in this corpus. The derived norms are necessarily in the corpus with the original ones. They are there although they have not been expressly promulgated. Their promulgation is concealed in the promulgation of other prescriptions (1963, pp. 157-158).

Esta idea se enlaza con la que ya apuntaba al final de la sección cuarta, la idea de que justificar una acción, desde el punto de vista normativo, requiere una cierta generalidad, requiere aducir una razón (o un conjunto de ellas) que decisivamente justifican realizar dicha acción en ese contexto. Tal vez no es otra cosa que una manifestación del principio leibniziano de razón suficiente, según el cual Nihil est sine ratione. En palabras de Alchourrón y Bulygin:

... el ideal de completitud normativa, esto es, la exigencia de que los sistemas normativos sean completos en el sentido de que solucionen todos los casos, presupone que todos los casos son solucionables. (...) Decir que todos los casos son solucionables significa que para todo caso hay o es posible construir un sistema normativo tal, que lo correlacione con alguna solución. La hipótesis 
José Juan Moreso

de casos no solucionables, es decir, situaciones en las que no hay razones (ni puede haberlas) para elegir tal o cual curso de acción, situaciones en las que toda elección es esencialmente arbitraria, es tan irracional, tan intolerable para la razón, como la idea de fenómenos inexplicables (fenómenos no causados por nada) (1971, pp. 171-172).

Obviamente, ello debe ser compatible con no considerar, como creo que nadie considera ahora, que la aplicación del derecho es una actividad mecánica que puede ser reducida a un algoritmo. Recordemos este pertinente comentario de Wittgenstein (1953, sec. 69) cuando se refiere al parecido de familia y usa los juegos como ejemplo y que, tal vez, está de acuerdo con la sugerencia de Bernard Williams en la cita que abre estos comentarios:

Someone says to me: "Shew the children a game". I teach them gaming with dice, and the other says "I didn't mean that sort of game". Must the exclusion of the game with dice have come before his mind when he gave me the order?

Sea como fuere, los trabajos de Luís Duarte d'Almeida agudizan nuestra visión de los problemas y, como es mi caso, aún si persistimos en defender la concepción ortodoxa, deberemos hacerlo con mayor cuidado, muy atentos a sus objeciones y argumentos.

\section{Bibliografía}

Aarnio, A. (1987). The Rational as Reasonable. A Treatise on legal Justification. Dordrecht: Reidel.

Alchourrón, C. E. y Bulygin, E. (1971). Normative Systems. New YorkWien: Springer.

Alexy, R. (1978). Theorie der juristischen Argumentation. Frankfurt a.M.: Suhrkamp.

Atienza, M. (2013). Curso de argumentación jurídica. Madrid: Trotta.

Beccaria, C. (1764/1981). Dei delitti e delle pene, a cura di F. Venturi, Torino: Einaudi. 
Aplicación del derecho y los casos Noara y Jaffa Cakes (en diálogo...

Bulygin, E. (2015). Essays in Legal Philosophy. C. Bernal, C. Huerta, T. Mazzarese, J.J. Moreso, P. Navarro y S. Paulson (eds.). Oxford: Oxford University Press.

Duarte d'Almeida, L. (2015). Allowing for Exceptions. A Theory of Defences and Defeasibility in Law. Oxford: Oxford University Press.

Duarte d'Almeida, L. (2019). On the Legal Syllogism. En Plunkett, D., Shapiro, S. J., Toth, K. (eds.), Dimensions of Normativity (pp. 335-364). Oxford: Oxford University Press.

Duarte d'Almeida, L. (2021). What Is it to Apply the Law? Law and Philosophy, 40, 361-386.

Ferrajoli, L. (1989). Diritto e ragione: teoria del garantismo penale. RomaBari: Laterza.

García Figueroa, A. (2009). Criaturas de la moralidad. Una aproximación neconstitucionalista al derecho a través de los derechos. Madrid: Trotta.

García Figueroa, A. (2012). Neoconstitucionalismo: dos (o tres) perros para un solo collar. Doxa, 34, 117-133.

Hernández Marín, R. (2019). Sobre el razonamiento principal de una sentencia judicial. Doxa, 42, 103-129.

Holton, R. (2002). Principles and Particularisms. Proceedings of the Aristotelian Society, Supplementary Volume 76, 191-210.

Maccormick, N. (1978). Legal Reasoning and Legal Theory. Oxford: Oxford University Press.

Mackeever, S. y Ridge, M. (2006). Principled Ethics. Generalism as a Regulative Ideal. Oxford: Oxford University Press.

Moreso, J. J. (2017). Moral Complications and Legal Structures. En M. Borowski, S.L. Paulson und J.R. Sieckmann (Hrsg.) Rechtsphilosophie und Grundrechtstheorie. Robert Alexys System (pp. 359-374). Tübingen: Mohr Siebeck.

Moreso, J. J. y Chilovi, S. (2018). Interpretive Arguments and the Application of the Law. En G. Bongiovanni, G. Postema, A. Rotolo, G. Sartor, C. Valentini, C. y D. Walton, (eds), Handbook of Legal Reasoning and Legal Argumentation (pp. 495-518). Dordrecht: Springer.

Raz, J. (1999). The Truth in Particularism. En J. Raz, Engaging Reason: On the Theory of Value and Action (pp. 218-246). Oxford: Oxford University Press. 
José Juan Moreso

Von Wright, G. H. (1963). Norm and Action. A Logical Enquiry. London: Routledge and Kegan Paul.

Williams, B. (1985). Ethics and the Limits of Philosophy. London: Fontana.

Wiggins, D. (2006). Ethics. Twelve Lectures on the Philosophy of Morality. London: Penguin.

Wittgenstein, L. (1953). Philosophical Investigations. Oxford: Basil Blackwell.

Wroblewski, J. (1974). Legal Syllogism and Rationality of Judicial Decision. Rechtstheorie, 5, 33-46. 